\title{
Pound of prevention, ounce of cure?
}

M

edical research aimed at identifying nascent disease is yielding some spectacular findings: findings that show, for example, that smaller and smaller nests of cancerous cells can be discovered earlier and earlier in the course of the disease; that asymptomatic atherosclerotic plaques in key vessels are identifiable earlier with noninvasive techniques; and that many degenerative diseases can be caught in their preclinical stages. Examples abound: colonoscopy for colonic polyps, mammography for breast cancer, CT scans for lung cancer and prostatespecific antigen (PSA) testing as a marker for prostate cancer risk. But is the effort worth it? Or are the immediate costs, both personal and societal, of earlier and earlier detection worth the benefits of gains in disease-free survival or life expectancy that will occur only much later - often decades later? What about the harms of early detection?

Take cancer of the prostate as an example. Before the development of PSA testing, most prostate cancer became manifest only as a coincidental finding on prostatic resection for benign prostatic hypertrophy, by the highly insensitive and nonspecific digital rectal examination, or because of metastases. Most detected tumours were at stages T2 or T3 and had high (more malignant) Gleason scores. With the advent of PSA measurements, clinicians are able to detect prostate cancer before it becomes symptomatic, such that most prostate cancers now detected are stage $\mathrm{T} 1$ and have lower Gleason scores. Indeed, very recent research shows that $15 \%$ of men with PSA levels lower than the current cut-off for biopsy ( $4 \mathrm{ng} / \mathrm{mL}$ ) harbour prostate cancer. ${ }^{1}$ Should these men undergo radical prostatectomy? No one knows. Should men with PSA values below the cut-off be told that 1 in 7 of them will have cancer? Yes. Will they demand biopsy? Some will. Even though we don't understand the natural history of these early malignant cells, will men with positive biopsy results instantly be labelled "sick" and begin to worry?

Undoubtedly.

The costs of secondary prevention of asymptomatic disease that are borne by patients and society are not trivial. Prolonged disease-free survival may well follow early extir- pation $^{2}$ but the results are not yet convincing, and the impact on quality of life still should be considered. ${ }^{3}$ And there are implications for health care resources. The benefits, harms and cost-effectiveness of early detection will require evaluation: large-scale randomized controlled trials will take years to fund (when they manage to obtain funding) and decades to complete and confirm. In the interim, patients (at least those with affordable access to the new tests and procedures) and their physicians will have to make their best guesses. The narcissism of Western societies leaves us in no doubt that our relentless pursuit of "health" and longevity will mean that more and more people in the land of the apparently well will obtain marginal diagnoses that gain them entry into the land of the officially sick.

Illich described this tendency as the "medicalization of life" and termed the result a "radical monopoly" by which medicine "reinforces a morbid society in which social control of the population by the medical system turns into a principal economic activity." "An unintended, underestimated and inevitable consequence of this radical monopoly is that more and more of our resources are spent on clinical procedures with untested cost-benefit assumptions, the costs being immediate and real, the benefits remote and uncertain. And the opportunity costs - whereby resources are deflected from other things, such as early childhood education, safer water, bike paths, culture and other aspects of happy and healthy living - will be large and not immediately perceptible. We need to proceed carefully and thoughtfully with each new means of medicalizing our lives. - CMAf

\section{References}

1. Thompson IM, Pauler DK, Goodman PJ, Tangen CM, Lucia MS, Parnes $\mathrm{HL}$, et al. Prevalence of prostate cancer among men with a prostate-specific antigen level $\leq 4.0 \mathrm{ng}$ per milliliter. N Engl 7 Med 2004;350(22):2239-46.

2. Siemens DR. Radical prostatectomy or watchful waiting in early prostate cancer? CMA7 2003;168(1):67.

3. Jewett MAS, Fleshner N, Klotz LH, Nam RK, Trachtenberg J. Radical prostatectomy as treatment for prostate cancer. CMAJ 2003;168(1):44-5.

4. Illich I. Limits to medicine. In: Medical nemesis: the expropriation of bealth. London (UK): Marion Boyars Publishers; 1976. p. 43. 